\title{
A!
}

This is an electronic reprint of the original article.

This reprint may differ from the original in pagination and typographic detail.

Kujala, J.; Slotte, J.; Tuomisto, F.; Hiller, D.; Zacharias, M.

\section{Si nanocrystals and nanocrystal interfaces studied by positron annihilation}

Published in:

Journal of Applied Physics

DOI:

$10.1063 / 1.4964503$

Published: 14/10/2016

Document Version

Publisher's PDF, also known as Version of record

Please cite the original version:

Kujala, J., Slotte, J., Tuomisto, F., Hiller, D., \& Zacharias, M. (2016). Si nanocrystals and nanocrystal interfaces studied by positron annihilation. Journal of Applied Physics, 120(14), 1-6. [145302].

https://doi.org/10.1063/1.4964503

This material is protected by copyright and other intellectual property rights, and duplication or sale of all or part of any of the repository collections is not permitted, except that material may be duplicated by you for your research use or educational purposes in electronic or print form. You must obtain permission for any other use. Electronic or print copies may not be offered, whether for sale or otherwise to anyone who is not an authorised user. 


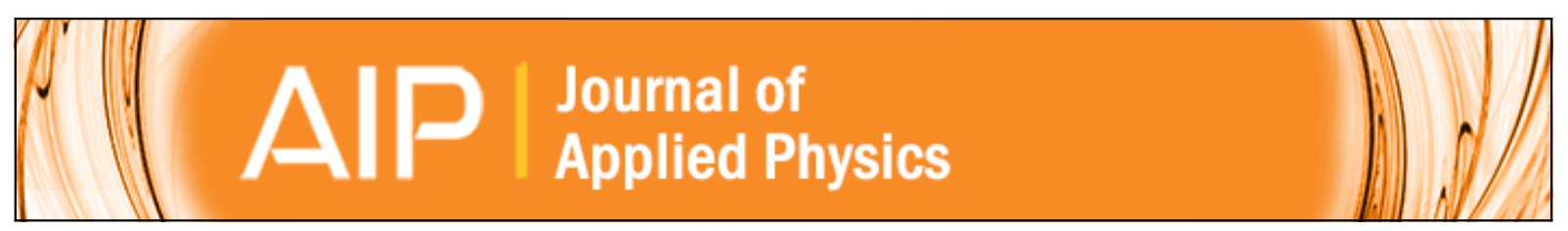

\section{Si nanocrystals and nanocrystal interfaces studied by positron annihilation}

J. Kujala, J. Slotte, F. Tuomisto, D. Hiller, and M. Zacharias

Citation: Journal of Applied Physics 120, 145302 (2016); doi: 10.1063/1.4964503

View online: http://dx.doi.org/10.1063/1.4964503

View Table of Contents: http://scitation.aip.org/content/aip/journal/jap/120/14?ver=pdfcov

Published by the AIP Publishing

\section{Articles you may be interested in}

Positron annihilation studies of the $\mathrm{AlOx} / \mathrm{SiO} 2 / \mathrm{Si}$ interface in solar cell structures

J. Appl. Phys. 111, 053515 (2012); 10.1063/1.3691895

Origin of cathodoluminescence from Si nanocrystal/ Si O 2 multilayers

J. Appl. Phys. 101, 034306 (2007); 10.1063/1.2433124

Stability of Se passivation layers on $\mathrm{Si}(001)$ surfaces characterized by time-of-flight positron annihilation induced Auger electron spectroscopy

J. Appl. Phys. 97, 103510 (2005); 10.1063/1.1897488

Elucidation of the surface passivation role on the photoluminescence emission yield of silicon nanocrystals embedded in $\mathrm{SiO} 2$

Appl. Phys. Lett. 80, 1637 (2002); 10.1063/1.1456970

Nanostructure of thin amorphous hydrogenated carbon films studied by positron annihilation and photoluminescence

J. Appl. Phys. 90, 404 (2001); 10.1063/1.1378063

\section{A $1 P$ Journal of Applied Physics}

INTRODUCING INVITED PERSPECTIVES Ultrafast magnetism and $\mathrm{THz}$ spintronics Authors: Jakob Walowski and Markus Münzenberg 


\title{
Si nanocrystals and nanocrystal interfaces studied by positron annihilation
}

\author{
J. Kujala, ${ }^{1}$ J. Slotte, ${ }^{1}$ F. Tuomisto, ${ }^{1}$ D. Hiller, ${ }^{2}$ and M. Zacharias ${ }^{2}$ \\ ${ }^{1}$ Department of Applied Physics, Aalto University School of Science, P.O. Box 14100, FI-00076 Aalto, Finland \\ ${ }^{2}$ IMTEK, Faculty of Engineering, Albert-Ludwigs-University Freiburg, Georges-Koehler-Allee 103, \\ 79110 Freiburg, Germany
}

(Received 23 June 2016; accepted 26 September 2016; published online 11 October 2016)

\begin{abstract}
$\mathrm{Si}$ nanocrystals embedded in a $\mathrm{SiO}_{2}$ matrix were studied with positron annihilation and photoluminescence spectroscopies. Analysis of the $S$ - and $W$-parameters for the sample annealed at $800^{\circ} \mathrm{C}$ reveals a positron trap at the interface between the amorphous nanodots and the surrounding matrix. Another trap state is observed in the $1150{ }^{\circ} \mathrm{C}$ heat treated samples where nanodots are in a crystalline form. Positrons are most likely trapped to defects related to dangling bonds at the surface of the nanocrystals. Passivation of the samples results on one hand in the decrease of the $S$-parameter implying a decrease in the open volume of the interface state and, on the other hand, in the strengthening of the positron annihilation signal from the interface. The intensity of the photoluminescence signal increases with the formation of the nanocrystals. Passivation of samples strengthens the photoluminescence signal, further indicating a successful deactivation of luminescence quenching at the nanocrystal surface. Strengthening of the positron annihilation signal and an increase in the photoluminescence intensity in passivated silicon nanocrystals suggests that the positron trap at the interface does not contribute to a significant extent to the exciton recombination in the nanocrystals. Published by AIP Publishing.
\end{abstract}

[http://dx.doi.org/10.1063/1.4964503]

\section{INTRODUCTION}

Bulk Si has very poor optical properties due to the indirect band gap. However, when the size of the crystal is in the nanometer scale, a strong quantum yield (QY) can be observed. Valenta et al. ${ }^{1}$ studied silicon quantum dots (SiQD) separated by $\mathrm{SiO}_{2}$ barriers. They reported an increase of QY in room temperature (RT) from $\sim 10 \%$ to $\sim 19 \%$ as a function of interlayer barrier thickness. In a study by Sangghaleh et al., ${ }^{2}$ photoluminescence (PL) from samples of colloidal and ligandpassivated SiQDs were measured. QY varied from 30\% to as high as $70 \%$. SiQD based light emitting diodes (LEDs) (e.g., Refs. 3 and 4) are not the only type of devices that benefit from the high QY. Due to its unique optical properties, SiQDs can as well be used as a sensing material. Nguyen et al. ${ }^{5}$ investigated dodecyl and amine-terminated SiQDs luminescence response to the presence of nitro benzene and dinitrotoluene (DNT). SiQD demonstrated a detection limit of $6 \mathrm{ppb}$ for DNT in the vapor phase. The detection limit for DNT in toluene solutions was on the order of a few hundred nM. The suitability of SiQDs to be used as a biomarker has also been studied. ${ }^{6}$ Organic dyes, used currently as bioimaging agents, can suffer from photobleaching, rendering them unsuitable for long-term imaging experiments. The research on the photostability of the SiQD (e.g., Refs. 7-9) indicated a more sustainable luminescence compared to those of the organic dyes.

In the case of crystalline $\mathrm{Si}$ quantum dots, or silicon nanocrystals ( $\mathrm{SiNC}$ ), embedded in $\mathrm{SiO}_{2}$ literature generally encompass five types of point defects, ${ }^{10}$ out of which three of them are unpaired electrons at dangling bond orbitals in crystalline $\mathrm{Si}$. $\mathrm{P}_{\mathrm{b}}$ and $\mathrm{P}_{\mathrm{b}(0)}$ centers represent the dangling bond centers at $\mathrm{Si}(111) / \mathrm{SiO}_{2}$ and $\mathrm{Si}(100) / \mathrm{SiO}_{2}$ interfaces, respectively. $\mathrm{P}_{\mathrm{b} 1}$ is similar to $\mathrm{P}_{\mathrm{b}}$ and $\mathrm{P}_{\mathrm{b}(0)}$ centers but with $\mathrm{a}$ strained Si-Si bond. Furthermore, these defects represent the non-radiative recombination paths that efficiently quench the photoluminescence (PL) from the respective quantum dot. $^{11,12}$

Positron annihilation spectroscopy (PAS) is well suited for studying the point defects in semiconductors, with selective sensitivity to negative and neutral vacancy defects and negatively charged ion-type defects. ${ }^{13}$ Positron methods are insensitive to the electrical conductivity of the material and can be easily applied to semiconductors regardless of the width of the band-gap. The ability to control the positron implantation energy allows for the study of defect distributions in thin layers and at interfaces. Kilpeläinen et al. ${ }^{14}$ used PAS to study interfaces between silicon nanocrystals and the $\mathrm{SiO}_{2}$ matrix. In that paper, superlattice with varying thicknesses was deposited on a $p$-type $\mathrm{Si}$ substrate by radio frequency (RF) magnetron sputtering. Samples subjected to a crystallization annealing and to a subsequent $\mathrm{H}_{2}$ passivation exhibited an enhancement in the PL signal suggesting that most of the interface defects were passivated with hydrogen. Mokry et al. ${ }^{15}$ studied silicon nanocrystal growth and location on thin $\mathrm{SiO} / \mathrm{SiO}_{2}$ films with $\mathrm{PL}$, transmission electron microscope (TEM), and PAS. In these experiments, Si nanocrystals were fabricated by implanting thermal $\mathrm{SiO}_{2}$ films and high temperature annealing. The results suggested that vacancies play a dominant role in facilitating silicon diffusion by which the growth of the nanocrystals proceeds. In this work, we study Si nanocrystals and their interfaces to the $\mathrm{SiO}_{2}$ matrix with a mono-energetic positron beam and PL. With the help of Doppler broadening measurements, a trap state at the interface of the nanocrystal and the surrounding $\mathrm{SiO}_{2}$ matrix is observed. Samples subjected to $\mathrm{H}_{2}$ treatment display increased PL intensity compared to unpassivated samples 
which indicates a decrease in the density of non-radiative PL-quenching centers.

\section{EXPERIMENTAL DETAILS}

The studied samples are multilayer structures consisting of $\mathrm{SiO}_{2} /$ Silicon Rich OxyNitride (SRON) bilayers deposited by plasma enhanced chemical vapor deposition (PECVD) on wet chemically cleaned $100 \mathrm{Si}$ and capped with $30 \mathrm{~nm} \mathrm{SiO}{ }_{2}$. Further details on the deposition process can be found in Ref. 16. A schematic of this structure is shown in Fig. 1. In the bilayers, the thickness of each $\mathrm{SiO}_{2}$ layer is $2 \mathrm{~nm}$, while the thicknesses of the $\mathrm{Si}$ rich layers are $1.5 \mathrm{~nm}, 2.5 \mathrm{~nm}$, $3.5 \mathrm{~nm}$, and $4.5 \mathrm{~nm}$. The number of bilayers was adjusted to maintain a total sample thickness of $\sim 250 \mathrm{~nm}$ (see Fig. 1). Additionally, a $250 \mathrm{~nm}$ stoichiometric $\mathrm{SiO}_{2}$ reference sample was fabricated. Each as-grown sample was subjected to one of the following annealings: $1 \mathrm{~h}$ at $800^{\circ} \mathrm{C}$ in $\mathrm{N}_{2}, 1 \mathrm{~h}$ at $1150^{\circ} \mathrm{C}$ in $\mathrm{N}_{2}, 1 \mathrm{~h}$ at $1150^{\circ} \mathrm{C}$ in $\mathrm{N}_{2}$, and subsequent $\mathrm{H}_{2}$ passivation at $500^{\circ} \mathrm{C}$ for $1 \mathrm{~h}$. Formation of amorphous nanodots is achieved via the $800{ }^{\circ} \mathrm{C}$ annealing, whereas annealing at $1150^{\circ} \mathrm{C}$ produces crystalline $\mathrm{Si}$ quantum dots.

Photoluminescence spectra of all samples were measured at room temperature using a Si-charge-coupled device (CCD) attached to a single grating monochromator and under excitation of a $\mathrm{HeCd}$ laser $(3.8 \mathrm{eV}$ line). All spectra were corrected for the spectral response of the setup.

A monoenergetic slow positron beam was used to study the positron annihilation characteristics in the multilayer $\mathrm{SiO}_{2} / \mathrm{SRON}$ samples. After implantation into the sample, positrons thermalize, diffuse, and ultimately annihilate with electrons. The diffusion length for the positron is typically tens to hundreds of nanometers depending on the material and types of defects and defect concentrations. Annihilation with electrons occurs while the positron is either in a delocalized state in the lattice or trapped at a defect or interface. In the annihilation event, two gamma quanta with an energy of $511 \mathrm{keV}$ are emitted in opposite directions. Small angular differences occur due to momentum conservation of the annihilating pair. In Doppler broadening spectroscopy, the broadening of the $511 \mathrm{keV}$ annihilation line due to the momentum distribution of the annihilating electron-positron

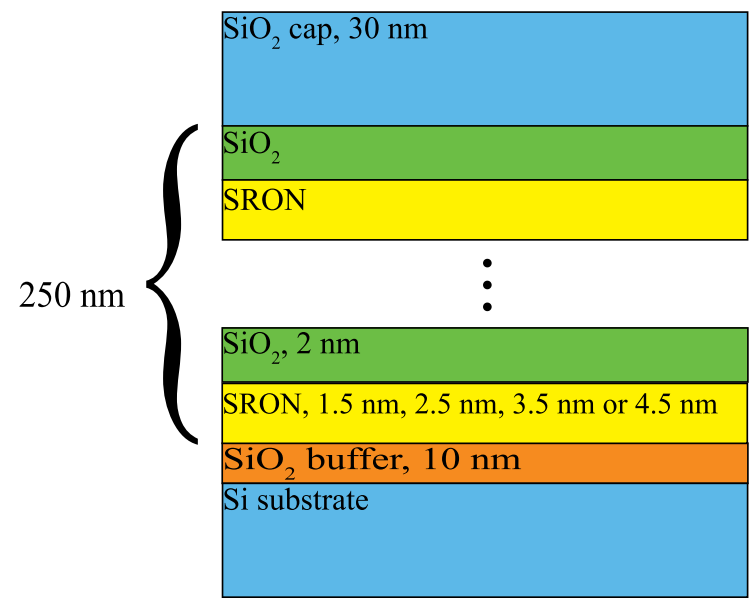

FIG. 1. Diagram for the sample structure, not in scale. pairs is detected. The shape of the Doppler broadened annihilation peak is typically described by line-shape parameters $S$ and $W$. The $S$-parameter is defined as the ratio of counts in the central, low momentum region, and the $W$ parameter describes the ratio of counts in the high momentum region of the Doppler spectrum. In this study, the $S$ region, which spans symmetrically across the $511 \mathrm{keV}$ peak, was set to $|E-511 \mathrm{keV}|<0.75 \mathrm{keV}$ (0.0-0.4 a.u). Correspondingly, the $W$ regions are defined on both sides of the peak at equal distances from the center. The $W$ region was set to 2.24 $\mathrm{keV}<\mid$ E-511 keV $\mid<5.00 \mathrm{keV}$ (1.2-2.7 a.u). In a typical Doppler broadening experiment, $S$ and $W$ parameters are measured as a function of positron implantation energy. The measured parameters are superpositions of the parameters for the annihilation states in the sample

$$
\begin{aligned}
S & =\sum_{\mathrm{i}} \eta_{\mathrm{i}} S_{\mathrm{i}}, \\
W & =\sum_{\mathrm{i}} \eta_{\mathrm{i}} W_{\mathrm{i}} .
\end{aligned}
$$

Here, $S_{\mathrm{i}}$ and $W_{\mathrm{i}}$ are the $S$ and $W$ parameters of the state $\mathrm{i}$, and $\eta_{\mathrm{i}}$ is the annihilation fraction in state $i$. These parameters can be presented in a $(S, W)$-plane which helps in annihilation state identification. In the simplest case, the possible positron annihilation states in the sample would be a defect free bulk state and a defect state. In such a case, a straight line between the states is expected in the $(S, W)$-plane. A deviation from this linearity suggests that more than two positron trapping states are present in the sample.

Both normal Doppler broadening and coincidence setups were used in this study. In the normal mode, one High-Purity $\mathrm{Ge}$ (HPGe) detector is used to measure the Doppler broadening of the $511 \mathrm{keV}$ annihilation line. In 1d-coincidence mode, one HPGe detector is used to measure the gamma emission from the positron-electron annihilations, whereas another detector is used as a gate. This type of setup reduces the background by one order of magnitude. Resolution of each detector was $1.25 \mathrm{keV}$ at $511 \mathrm{keV}$. The coincidence Doppler measurements were performed at RT. Approximately, $20 \times 10^{6}$ counts were collected for each coincidence spectrum. In the normal Doppler mode, positrons were implanted at RT with several acceleration voltages ranging from 0.3 to $25 \mathrm{kV}$ with $1 \times 10^{6}$ counts per Doppler spectrum. More details on the experimental technique can be found in Refs. 13 and 17.

\section{RESULTS}

Figure 2 shows the results from the PL measurements. Fig. 2(a) shows the PL spectra from the $3.5 \mathrm{~nm}$ sample at all four annealing stages. A large increase in the PL intensity is observed after the formation of the NCs at $1150{ }^{\circ} \mathrm{C} . \mathrm{H}_{2}$ treatment further increases the PL intensity, which is attributed to the passivation of dangling bond defects at the $\mathrm{SiNC} / \mathrm{SiO}_{2}-$ interfaces. ${ }^{12,18}$ Additionally, a small shift of PL peak towards higher wavelengths can be observed in the case of the passivated sample. This redshift is due to the emission enhancement from the larger nanocrystals of a given size distribution; larger nanocrystals are more prone to be affected by the non-radiative 


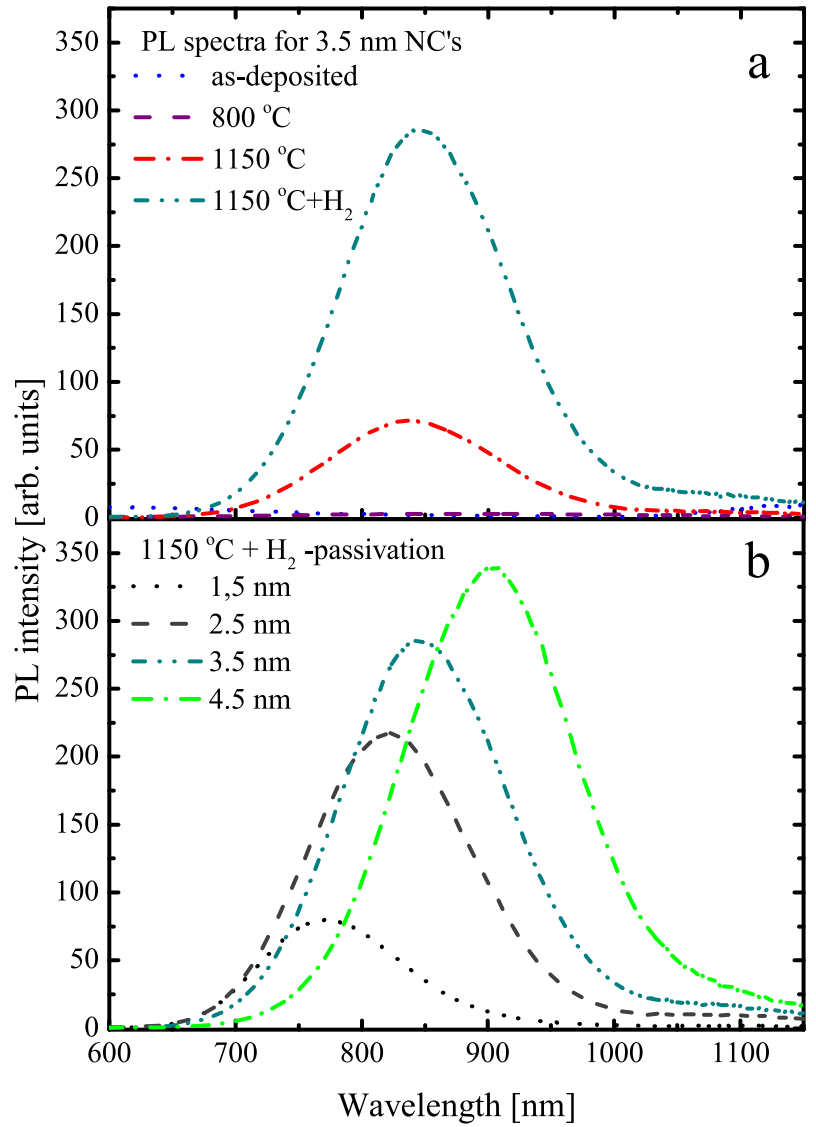

FIG. 2. Photoluminescence intensities as a function of wavelength. Shown are PL spectra from $3.5 \mathrm{~nm}$ SiNC samples after production step (a) and from passivated samples having varying nanocrystal sizes (b).

defects. ${ }^{12}$ Fig. 2(b) shows the spectra after the $1150{ }^{\circ} \mathrm{C}+\mathrm{H}_{2}$ passivation step. The effect of the quantum confinement, i.e., a blue shift of the PL peak with decreasing NC size, is clearly observed.

Fig. 3 presents the normalized $S$ - and $W$-parameters for the $3.5 \mathrm{~nm}$ sample as a function of positron implantation energy after different annealings. In our study, the line-shape parameters were normalized to the parameters from a defect free $\mathrm{Si}$ sample with $S_{\mathrm{Si}}=0.5515$ and $W_{\mathrm{Si}}=0.0175$. The gray full lines in the figures correspond to the $S$ - and $W$-parameters for defect free Si. Positron implantation can be described with a Makhovian implantation profile for slow positrons. ${ }^{19}$ The width of the profile is dependent on the acceleration energy, i.e., the larger the energy, the broader is the profile. The maximum in the $S$-parameter at $3.5 \mathrm{keV}$ for the asdeposited sample is an indication of the positron implantation profile being centered in the layer. Due to the nature of the distribution profile, the information depth, i.e., the range under which $95-97 \%$ of all of the annihilations occur, is two times the mean stopping depth of positrons. In the case of $3.5 \mathrm{keV}$ implantation energy, the information depth would reach from the surface to $\sim 300 \mathrm{~nm}$. Amorphous nanodots are formed as a result of $800{ }^{\circ} \mathrm{C}$ annealing. The $S$-parameter of the layer decreases compared to the as-deposited sample. This change in the $S$-parameter suggests that the annihilation state is different in annealed samples. In the $1150{ }^{\circ} \mathrm{C}$ heat treatment, crystalline nanodots are formed. The $S$-parameter

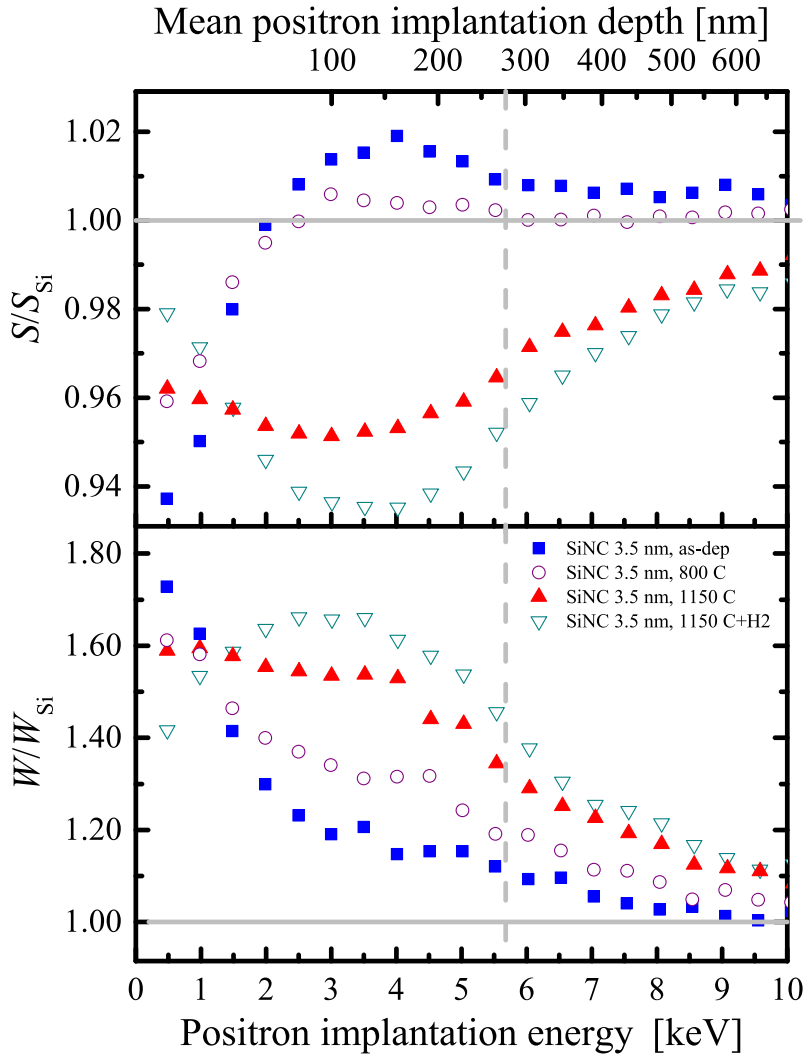

FIG. 3. Normalized $S$ - and $W$-parameters as a function of positron implantation energy. Full lines represent the corresponding parameters for bulk Si. The dashed line denotes the interface between the deposited layer and the substrate.

of the layer is $\sim 5 \%$ lower compared to that of the $800^{\circ} \mathrm{C}$ heat treated sample. Passivation further decreases the $S$-parameter.

In Fig. 4 , the normalized $(S, W)$-plots with different $\mathrm{NC}$ sizes and heat treatments are shown. Arrows in the figure indicate the direction of the increasing positron implantation energy. Added to the graphs is the $\mathrm{SiO}_{2} / \mathrm{Si}$ interface point reported earlier by Kauppinen et al. ${ }^{20}$ Three positron annihilation states can be distinguished in the plots and are denoted as follows: surface (Sf), layer (L), and substrate (S). After the formation of nanocrystals, the layer states (circled in the figure) are close to the $\mathrm{SiO}_{2} / \mathrm{Si}$-interface point. The passivation of the samples leads to yet a stronger signal from the layer which is seen as sharp turning points at the intersection of the surface-layer and the layer-bulk lines. The changes seen in the $(S, W)$-plots, shown in Fig. 4, are predominantly due to changes in the nanocrystals and not in the surrounding matrix. In the SiNC samples, the $S$-parameter decreases with each production step, whereas an opposite behavior can be seen in $\mathrm{SiO}_{2}$ (see inset in Fig. 4(b)). Thus, the evolution of the line-shape parameters is clearly different in the SiNC samples compared to $\mathrm{SiO}_{2}$.

Ratio spectra for the $3.5 \mathrm{~nm}$ sample are presented for different heat treatments in Fig. 5 measured with an acceleration voltage of $3.5 \mathrm{keV}$. With this acceleration voltage, the average positron implantation depth is at the middle of the nanocrystal layer. The resulting Doppler spectra were divided with a defect free Si reference spectrum. The intensity at low momenta $(0<p \leq 0.4$ a.u. in atomic units $)$ 


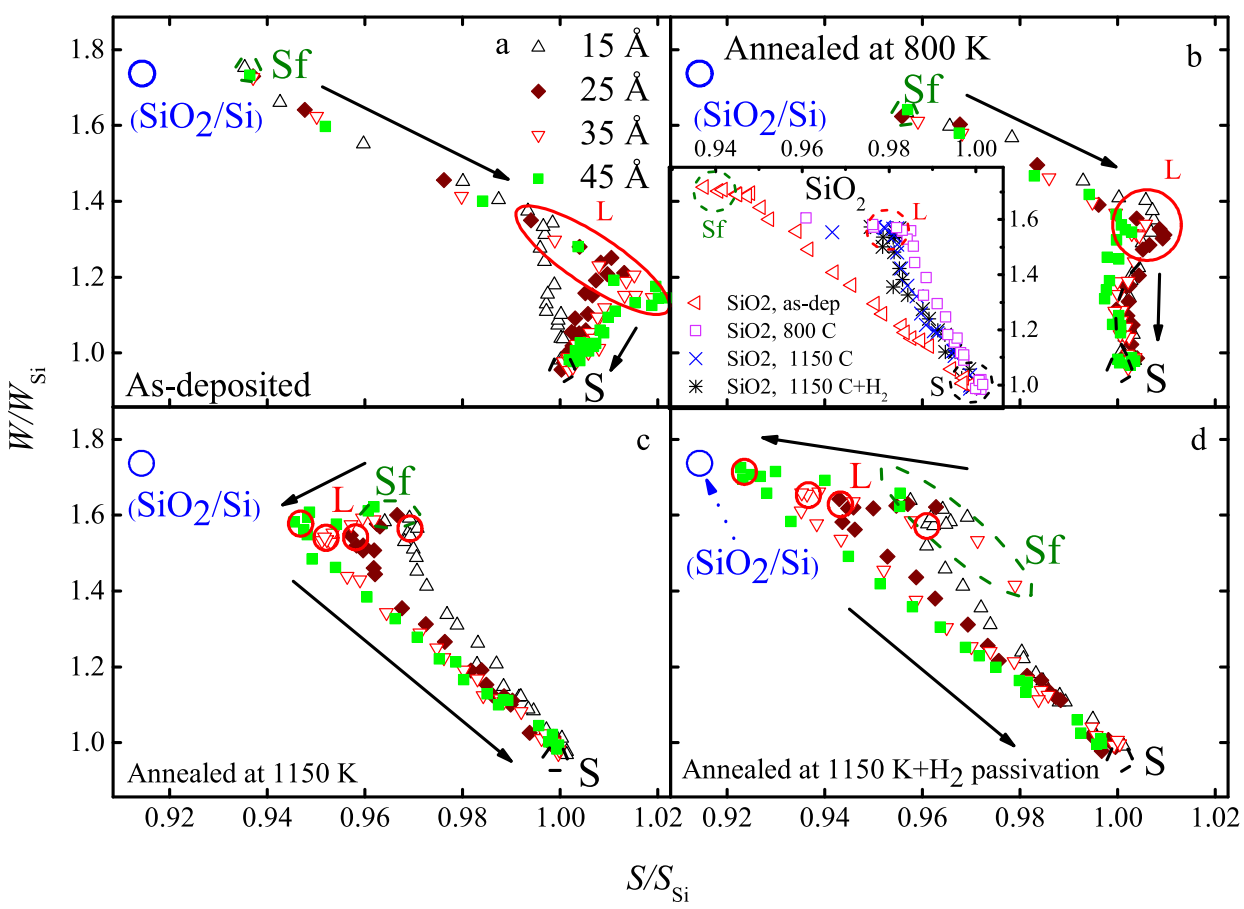

FIG. 4. Normalized $S(W)$-plots of different SiNC sizes and heat treatments. Arrows in the figure indicate the increasing positron implantation energy. Surface (Sf), substrate (S), layer (L), and $\mathrm{SiO}_{2} / \mathrm{Si}$ interface point ${ }^{20}$ are denoted in the plots. In the inset, normalized $S(W)$-plots of $\mathrm{SiO}_{2}$ after different heat treatments are presented. Evolution of line-shape parameters contrasts to that of SiNC. This suggests that the effects observed in the $(S, W)$ plots for SiNC are due to changes in the SiNC and not in the lattice.

corresponds to positrons annihilating mainly with valence electrons, while the intensity at high momenta ( $p>1.4$ a.u.) corresponds to positrons annihilating mainly with core electrons. The window used for the determination of $W$-parameter is indicated in the figure. The defining feature that all spectra share is a peak at $\sim 1.5$ a.u. The lowest peak is seen in the as-deposited sample. The increase in the peak height is seen after $800{ }^{\circ} \mathrm{C}$ and $1150{ }^{\circ} \mathrm{C}$ treatments, whereas the passivation step slightly decreases the intensity of the peak.

\section{DISCUSSION}

As mentioned in Section III, the implantation profile for positrons deposited with $3.5 \mathrm{keV}$ is centered in the bilayer

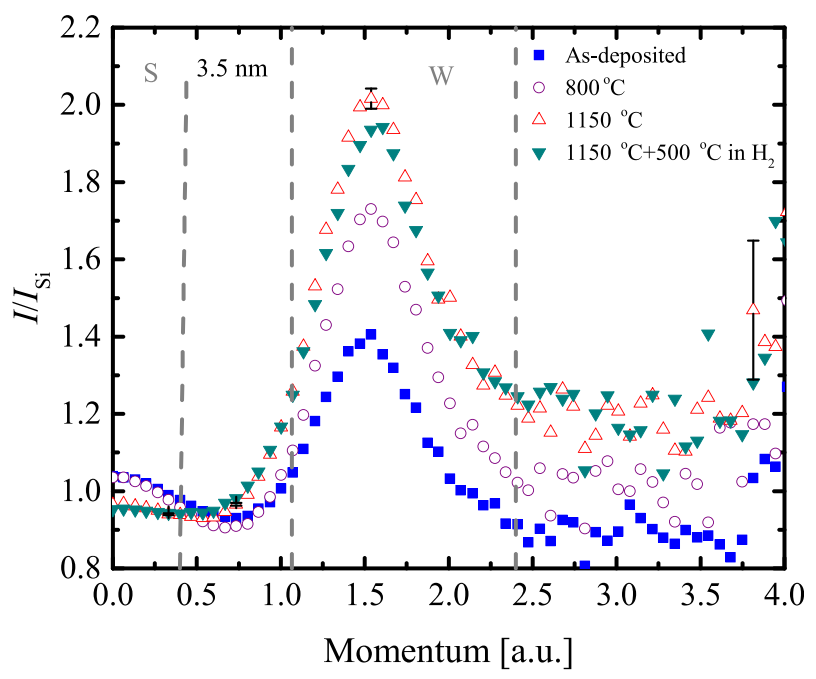

FIG. 5. Ratio spectra from $3.5 \mathrm{~nm}$ sample measured after various production steps. Positrons were implanted with the energy of $3.5 \mathrm{keV}$. Dashed lines denote the window used in determining the $W$-parameter value in the analyses. A few representative error-bars are added to the plot to indicate the accuracy of the measurements at different momenta. A defect free bulk Si sample was used as a reference. Measurements were performed at room temperature. stack. The highest $S$-value is in the as-deposited sample, and this value is clearly above the solid line describing annihilations in defect free Si bulk. Each production step decreases the $S$-parameter in the layer, while at the surface, an increase in the parameter can be observed. Steep slopes in the $S$ parameters at $\sim 0.5$ to $\sim 3.5 \mathrm{keV}$ for $800^{\circ} \mathrm{C}$ and passivated samples and at 3.5 to $7.5 \mathrm{keV}$ for sample treated at $1150{ }^{\circ} \mathrm{C}$ and the passivated sample imply that the trap state in each respective sample is strong, and thus, positrons are trapped quickly after the thermalization. Clear maxima and minima in the $S$-parameter at $3.5 \mathrm{keV}$ for the as-deposited and passivated samples indicate that rather than annihilating at delocalized states, positrons are localized to this strong interface trap in the deposited layer.

The positron states and their dependence on the process step can be seen from the $(S, W)$-plots in Fig. 4. During $800^{\circ} \mathrm{C}$ heat treatment (panel b in Fig. 4), Si atoms from the SRON layer diffuse into the $\mathrm{SiO}_{2}$ lattice and form amorphous Si clusters. This leads to changes in the $S$ - and $W$ parameters in the layer. One of the changes is the appearance of a small curvature (denoted by a dashed line) seen in the line between the layer state and the surface state, which indicates a modification of $S$ - and $W$-parameters by a new annihilation state related to the amorphous nanodots. This annihilation state is most likely either related to the dangling bond defects at the amorphous nanodots, or the interface itself will trap positrons and cause the localized annihilation state. In the case of $3.5 \mathrm{~nm}$ and $4.5 \mathrm{~nm}$, NC samples annealing at $1150{ }^{\circ} \mathrm{C}$ for one hour (panel c) produce sharp turning points in the $(S, W)$-plots close to the $\mathrm{Si} / \mathrm{SiO}_{2}$ interface point, whereas for smaller $\mathrm{NC}$, this turning point is not as sharp. The area of the interface between NC and the surrounding matrix depends on the size of the NC. Larger interface area can act as a strong trap and lead to a sharp turning point seen in the $(S, W)$-plots. Correspondingly, the surfaces of smaller $\mathrm{NCs}$ are not large enough to result in a sharp turning point in 
the $S W$-plane. With the formation of crystalline nanodots, a large reduction in the $S$-parameter is observed (see Fig. 3 and panel d in Fig. 4). This suggests, on one hand, a change in the annihilation environment and on the other hand, a change in the average open volume at the interface. Hydrogen passivation of the samples aims to compensate the $\mathrm{Si}$ dangling bond defects at the interface by forming $\mathrm{Si}-\mathrm{H}$ bonds. At the same time, the $S$-parameter is further reduced. In this case, a strong increase in the PL intensity of the $35 \AA$ sample can be seen, indicating an increase in the light conversion efficiency.

The Doppler broadening peak can be divided into two parts. Positrons annihilating with high momentum core electrons form the broad low intensity part of the annihilation peak. Consequently, positron annihilations with low momentum electrons (mostly valence electrons) give rise to a narrow and high intensity part of the annihilation peak. The Doppler peak is a sum of these two parts. The defect free Si reference sample contains a kink at momenta where the intensities of the high and low positron-electron momentum distributions are equal, i.e., $\mathrm{I}(p)_{\text {core }}=\mathrm{I}(p)_{\text {valence. }}$. When vacancy defects are present in the lattice, the translational symmetry of the lattice is disturbed. This is seen in the Doppler spectrum as a smoothing of the kink. ${ }^{21}$ Consequently, a peak is observed in the $I / I_{\mathrm{Si}}$-ratio at the corresponding momentum. This type of peak was observed in earlier studies on $p$-type Si, e.g., in Refs. 22 and 23 and was associated with the presence of the oxygen-vacancy related traps in the sample. Yet, as mentioned above, the smoothing of the kink occurs when the translational symmetry of the lattice is broken and does not require the presence of oxygen in the sample. Thus, the annihilation peak shown in Fig. 5 for the SiNC samples may have other origins than the positron trapping to O-V-type traps. Sharp turning points in $(S$, $W$ )-plots for the passivated SiNC and large peaks in momentum ratio curves indicate a strong localized positron annihilation state at the interface. An increase in the PL intensity and the strengthening of the positron annihilation signal after the passivation of the samples suggest that this annihilation state is not detrimental to the light conversion efficiency of SiNC. In the study by Kilpeläinen et al., a correspondence between the PL intensity and defects were identified, i.e., the PL signal originated from the defects at the surface of the nanocrystals. In the case of PECVD grown samples, the quantum confinement effect can be observed. It is likely that in both samples (RF sputtered and PECVD grown), the annihilation states observed by PAS are related to dangling bond defects formed at the interface between the nanocrystal and the surrounding lattice. ${ }^{10,18}$ On the other hand, the observed quantum confinement in this study clearly shows that PAS and PL measure different phenomena in contrast to the earlier study by Kilpeläinen $e t$ al.

As the atomic structures of $\mathrm{Si}$ and Ge are very similar, it is worth to note the studies by Madia et al. ${ }^{24,25}$ in which $\mathrm{GeP}_{\mathrm{b} 1}$ centers at interfaces of $\mathrm{SiO}_{2} / \mathrm{Ge}_{\mathrm{x}} \mathrm{Si}_{1-\mathrm{x}} / \mathrm{SiO}_{2}$ heterostructures were observed with PAS. A decrease in the positron trapping efficiency of these dangling bond defects was observed there with hydrogen passivation. On the contrary, in our SiNC samples, the positron trapping efficiency of the defects at the interface increases with passivation, indicating a clear difference in electronic activities of oxide interface defects related to $\mathrm{Si}$ and $\mathrm{Ge}$.

\section{CONCLUSIONS}

In conclusion, we studied the defect centers in silicon quantum dots embedded in $\mathrm{SiO}_{2}$ matrix. As-deposited samples consisted of $\mathrm{SRON} / \mathrm{SiO}_{2}$ bilayers that were annealed to induce the SRON-phase separation and Si-clustering $\left(800^{\circ} \mathrm{C}\right)$ and, finally, crystallization $\left(1150^{\circ} \mathrm{C}\right)$. A subsequent $\mathrm{H}_{2}$ treatment was carried out to passivate dangling bond interface defects. Analysis of the $S$ - and $W$-parameters of the PAS spectra for the sample heated at $800^{\circ} \mathrm{C}$ reveals a positron trap at the interface between the amorphous nanodots and the surrounding matrix. Another trap state is observed in the $1150{ }^{\circ} \mathrm{C}$ heat treated samples, where nanodots are in a crystalline form. The change in the line-shape parameters is related to the passivation of the dangling bond defects at the NC surface. The intensity of the PL signal increases with the formation of the nanocrystals. Strongest PL is measured from the passivated sample. This indicates a successful compensation of dangling bond defects in the interface between $\mathrm{Si}$ nanocrystal and $\mathrm{SiO}_{2}$ lattice. Strengthening of the positron annihilation signal and an increase in the PL intensity in passivated SiNC suggest that the positron trap at the interface does not contribute to a significant extent to the exciton recombination in the nanocrystals.

${ }^{1}$ J. Valenta, M. Greben, S. Gutsch, D. Hiller, and M. Zacharias, Appl. Phys. Lett. 105, 243107 (2014).

${ }^{2}$ F. Sangghaleh, I. Sychugov, Z. Yang, J. G. Veinot, and J. Linnros, ACS Nano 9, 7097 (2015).

${ }^{3}$ R. J. Anthony, K.-Y. Cheng, Z. C. Holman, R. J. Holmes, and U. R. Kortshagen, Nano Lett. 12, 2822 (2012).

${ }^{4}$ F. Maier-Flaig, J. Rinck, M. Stephan, T. Bocksrocker, M. Bruns, C. Kubel, A. K. Powell, G. A. Ozin, and U. Lemmer, Nano Lett. 13, 475 (2013).

${ }^{5}$ A. Nguyen, C. M. Gonzalez, R. Sinelnikov, W. Newman, S. Sun, R. Lockwood, J. G. Veinot, and A. Meldrum, Nanotechnology 27, 105501 (2016).

${ }^{6}$ B. F. McVey and R. D. Tilley, Acc. Chem. Res. 47, 3045 (2014).

${ }^{7}$ A. Shiohara, S. Prabakar, A. Faramus, C.-Y. Hsu, P.-S. Lai, P. T. Northcote, and R. D. Tilley, Nanoscale 3, 3364 (2011).

${ }^{8} \mathrm{Z}$. Li and E. Ruckenstein, Nano Lett. 4, 1463 (2004).

${ }^{9}$ J. H. Warner, A. Hoshino, K. Yamamoto, R. Tilley et al., Angew. Chem. 117, 4626 (2005).

${ }^{10}$ A. Stesmans, M. Jivanescu, S. Godefroo, and M. Zacharias, Appl. Phys. Lett. 93, 023123 (2008).

${ }^{11}$ C. Delerue, G. Allan, and M. Lannoo, Phys. Rev. B 48, 11024 (1993).

${ }^{12}$ D. Hiller, M. Jivanescu, A. Stesmans, and M. Zacharias, J. Appl. Phys. 107, 084309 (2010).

${ }^{13}$ F. Tuomisto and I. Makkonen, Rev. Mod. Phys. 85, 1583 (2013).

${ }^{14}$ S. Kilpeläinen, J. Kujala, F. Tuomisto, J. Slotte, Y.-W. Lu, and A. N. Larsen, J. Appl. Phys. 114, 164316 (2013).

${ }^{15}$ C. R. Mokry, P. J. Simpson, and A. P. Knights, J. Appl. Phys. 105, 114301 (2009).

${ }^{16}$ A. Hartel, D. Hiller, S. Gutsch, P. Löper, S. Estradé, F. Peiró, B. Garrido, and M. Zacharias, Thin Solid Films 520, 121 (2011).

${ }^{17}$ R. Krause-Rehberg and H. S. Leipner, Positron Annihilation in Semiconductors: Defect Studies (Springer Science and Business Media, Germany, 1999), Vol. 127.

${ }^{18}$ M. Zacharias, D. Hiller, A. Hartel, and S. Gutsch, Phys. Status Solidi A 209, 2449 (2012).

${ }^{19}$ P. Asoka-Kumar and K. Lynn, Appl. Phys. Lett. 57, 1634 (1990). 
${ }^{20}$ H. Kauppinen, C. Corbel, L. Liszkay, T. Laine, J. Oila, K. Saarinen, P. Hautojärvi, M. Barthe, and G. Blondiaux, J. Phys. Condens. Matter 9, 10595 (1997).

${ }^{21}$ A. C. Kruseman, Two-Dimensional ACAR and Low-Background DBAR Studies on Materials With Defects (TU Delft, Delft University of Technology, The Netherlands, 1999).

${ }^{22}$ R. Brusa, W. Deng, G. Karwasz, A. Zecca, and D. Pliszka, Appl. Phys. Lett. 79, 1492 (2001).
${ }^{23}$ A. Kruseman, H. Schut, A. Van Veen, and M. Fujinami, Nucl. Instrum. Methods Phys. Res., Sect. B 148, 294 (1999).

${ }^{24}$ O. Madia, A. Nguyen, N. Thoan, V. Afanasev, A. Stesmans, L. Souriau, J. Slotte, and F. Tuomisto, Appl. Surf. Sci. 291, 11 (2014).

${ }^{25}$ O. Madia, N. Segercrantz, V. Afanas' ev, A. Stesmans, L. Souriau, J. Slotte, and F. Tuomisto, Phys. Status Solidi B 251, 2211 (2014). 